\title{
Progresión de talla y peso en niños y niñas entre 6 y 12 años y su diferencia con las tablas de Ramos Galván 40 años después
}

\author{
Aldo Ferreira-Hermosillo, ${ }^{1}$ Ivonne Roy-García, ${ }^{2}$ Rodolfo Rivas-Ruiz, ${ }^{2}$ Juan José Palacios-Butchard, ${ }^{3}$ \\ Moisés Mercado y Juan O. Talavera * \\ ${ }^{1}$ Instituto Mexicano del Seguro Social, Centro Médico Nacional Siglo XXI, Unidad de Investigación Médica en Enfermedades Endocrinas; ${ }^{2}$ Instituto \\ Mexicano del Seguro Social, Centro Médico Nacional Siglo XXI, Centro de Adiestramiento en Investigación Clínica; ${ }^{3}$ Programa Nacional de \\ Activación Física Ponte al 100; ${ }^{4}$ Centro Médico ABC, Departamento de Enseñanza e Investigación. Ciudad de México, México
}

\section{Resumen}

Introducción: El peso y la talla de niños y adolescentes son un reflejo del estado de salud y desarrollo socioeconómico de la población. Objetivo: Evaluar las progresiones de talla y peso de niños y niñas mexicanos y compararlas con las tablas del doctor Ramos Galván a 40 años de distancia. Método: Encuesta transversal realizada en población del Programa Nacional de Activación Física Ponte al 100, que incluye niños y niñas de seis a 12 años. Resultados: Se evaluaron 43670 niños y 44103 niñas, que se estratificaron por sexo y edad. La progresión de talla entre los seis y 12 años fue de $21 \mathrm{~cm}$ en hombres y de $22 \mathrm{~cm}$ en mujeres; la progresión de peso fue de 9.86 y $10.05 \mathrm{~kg}$, respectivamente para hombres y mujeres. La proporción de niños de seis y 12 años con sobrepeso fue de 11.2 y 9 \% y con obesidad, de 14.7 y $15 \%$. La proporción de niñas de seis y 12 años con sobrepeso fue de 8.2 y $9.1 \%$ y con obesidad, de 21.7 y $13.3 \%$, respectivamente. Al comparar los valores obtenidos con los de las tablas del doctor Ramos Galván para niños y niñas, el promedio de diferencia fue de $2 \mathrm{~cm}$. Conclusiones: No se documentó un incremento secular de la talla ni del peso en los últimos 40 años.

PALABRAS CLAVE: Peso. Talla. Tablas de crecimiento. Crecimiento. Incremento secular de la talla.

\section{Height and weight progression patterns in Mexican children aged between 6 and 12 years and differences with Ramos-Galvan growth charts 40 years later}

\begin{abstract}
Introduction: Children and adolescents weight and height are a reflection of the health status and socioeconomic development of a population. Objective: To evaluate height and weight progression patterns of Mexican children and compare them with Dr. Ramos-Galván growth charts 40 years later. Method: Cross-sectional survey conducted on the population of the National Physical Activation Program Ponte al 100, which includes boys and girls aged 6-12 years. Results: 43,670 boys and 44,103 girls were assessed, stratified by gender and age. The height progression pattern between six and 12 years was $21 \mathrm{~cm}$ in males and $22 \mathrm{~cm}$ in females, whereas the weight progression pattern was 9.86 and $10.05 \mathrm{~kg}$, respectively, for males and females. The proportion of 6- and 12-year-old boys who were overweight was 11.2 and $9 \%$, while 14.7 and $15 \%$ were obese. The proportion of 6- and 12-year-old girls who were overweight was 8.2 and $9.1 \%$, whereas 21.7 and $13.3 \%$, respectively, were obese. When the obtained values were compared with those of Dr. Ramos Galván growth charts for boys and girls, the average difference was $2 \mathrm{~cm}$. Conclusions: No secular height or weight increase within the last 40 years was documented.
\end{abstract}

KEY WORDS: Weight. Height. Growth charts. Growth. Secular height increase. 


\section{Introducción}

Si bien la modernización y globalización de los países en desarrollo es responsable, parcialmente, de problemas sociales y económicos, también ha resultado en importantes mejoras en la calidad de vida como incremento en el acceso y distribución de los alimentos, urbanización, educación y salud, que han redundado en reducción de la morbimortalidad infantil y mejora nutricional. ${ }^{1-4}$ Portugal refiere un incremento en la talla de $5.7 \mathrm{~cm}$ en 40 años $^{5} \mathrm{y}$ en algunos países latinoamericanos se ha registrado un incremento de $9 \mathrm{~cm}$ en la talla final del adulto. ${ }^{6,7}$

La atención médica del niño sano se basa en la vigilancia de la talla y el peso. En México, las últimas estandarizaciones de talla fueron realizadas por el doctor Ramos Galván en 1975, con predominio de niños de la Ciudad de México, quienes tenían grandes ventajas de nutrición en relación con la población rural. ${ }^{8,9}$ De igual forma, se han recomendado las tablas de talla y peso de los Centros para el Control de Enfermedades de Estados Unidos (CDC, Centers for Disease Control), publicadas en el $2000 .{ }^{10}$ El propósito es contar con parámetros actualizados de seguimiento del niño sano mexicano y determinar si en la población mexicana ha existido un incremento en la talla en los últimos 40 años, considerando zonas rurales y urbanas. ${ }^{11,12}$

\section{Métodos}

El presente estudio es un análisis secundario de Ponte al $100,{ }^{13}$ programa nacional de intervención llevado a cabo en escuelas primarias y secundarias públicas de México, en el que se prescribe de manera individualizada un régimen de ejercicio físico y de alimentación. En cada escuela participante se realiza una reunión informativa a la que acuden voluntariamente alumnos, padres de familia y personal docente. A los profesores que acepten participar como evaluadores se les imparte un curso en el que se les instruye en el uso de las técnicas de medición antropométrica y de capacidad funcional, así como en el registro de los datos en una plataforma informática. Los alumnos cuyos padres o tutores acepten participar mediante la firma del consentimiento informado correspondiente son sometidos a una evaluación inicial que incluye lo siguiente:

- Registro de datos demográficos (edad, sexo, lugar de origen y de residencia).
- Antropometría y composición corporal (peso, taIla, índice de masa corporal, porcentaje de grasa corporal y de masa magra, circunferencia de cintura, cadera y brazo).

- Aptitud motora (fuerza muscular y flexibilidad de brazo, pierna y abdomen).

- Aptitud musculoesquelética (equilibrio, velocidad y agilidad).

- Aptitud cardiorrespiratoria (consumo máximo de oxígeno durante una prueba de carrera de $20 \mathrm{~m}$ ).

- Aptitud neuropsicológica (memoria y atención evaluadas mediante una prueba estándar de desafío de memoria).

Hasta ahora, el programa ha inscrito a más de un millón de niños y adolescentes y actualmente se están analizando los datos sobre el resultado de la intervención nutricional y de ejercicio. Para el presente estudio se utilizaron los datos demográficos y de composición corporal tomados durante la evaluación inicial. Se excluyó a los niños con alguna enfermedad conocida, a quienes usaran fármacos en forma crónica, estuviesen en algún programa de nutrición especial o mostraran alguna alteración en la medición de signos vitales en reposo, en ese caso se les refirió al centro de salud local para recibir atención médica.

La altura de pie se midió con un estadiómetro portátil, de acuerdo con las pautas antropométricas estándar, con el participante de pie en plano de Frankfurt. El peso total y el porcentaje de grasa corporal se determinaron mediante análisis de impedancia bioeléctrica (Tanita UM-081 ${ }^{\circledR}$, División Internacional de Tanita, Reino Unido). El índice de masa corporal se calculó mediante la fórmula peso/talla ${ }^{2}$. Estos datos son almacenados en una plataforma informática a la cual cada participante puede acceder solamente a sus propios datos por medio de un nombre de usuario y contraseña. Solo los miembros del equipo Ponte al 100 tienen ingreso a los datos de todos los participantes, en apego a las leyes de confidencialidad.

El proyecto de Ponte al 100 fue aprobado por el Comité de Investigación y Ética del Centro Médico American British Cowdray de la Ciudad de México.

Se calculó la media para talla y peso de cada grupo de edad con su desviación estándar y rango. A partir de estos valores se realizaron las curvas de crecimiento por sexo y edad. Finalmente, por sexo se compararon las medias de talla con las consignada en las tablas del doctor Ramos Galván de 1975 y las de los CDC del año 2000, a través de metaanálisis en red para estudios transversales ${ }^{14}$ para medias ponderadas con efectos aleatorios; se utilizó el programa 
RevMan 5 de Colaboración Cochrane. Los resultados fueron graficados usando forest plots con diferencias de medias e intervalos de confianza de $95 \%$.

\section{Resultados}

Se analizaron datos antropométricos de 43670 niños y 44103 niñas entre los seis y 12 años. En las Tablas 1 y 2 se muestran los valores medios de estatura en metros (Figura 1), peso en kilogramos, índice de masa corporal (IMC), porcentaje de grasa, perímetro de cintura en $\mathrm{cm}$ y su clasificación en sobrepeso y obesidad de acuerdo con los criterios de la agencia CDC.

La progresión de talla de los seis a 12 años fue de $21 \mathrm{~cm}$ en los hombres $(1.20 \pm 0.10$ a $1.41 \pm 0.07)$ y $22 \mathrm{~cm}$ en las mujeres $(1.19 \pm 0.11$ a $1.41 \pm 0.07)$. La progresión del peso fue de $9.86 \mathrm{~kg}$ en los hombres $(25.62 \pm 6.87$ y $35.48 \pm 7.33)$ y $10.05 \mathrm{~kg}$ en las mujeres $(25.42 \pm 6.63$ a $35.47 \pm 7.56)$. La progresión del IMC fue de $0.8 \mathrm{~kg} / \mathrm{m}^{2}$ en los hombres $(17.1 \pm 2.7 \mathrm{a}$ $17.9 \pm 2.8)$ y $0.4 \mathrm{~kg} / \mathrm{m}^{2}$ en las mujeres $(17.4 \pm 3.4 \mathrm{a}$ $17.8 \pm 2.8)$.

El porcentaje de grasa corporal varió entre $16.03 \pm 7.9$ y $18.79 \pm 8.5$ en hombres y entre $19.2 \pm 7.2$ y $21.48 \pm 7.0 \%$ en mujeres. El perímetro de cintura en hombres fue de $58.4 \pm 8.02 \mathrm{~cm}$ a $68.18 \pm 9.57 \mathrm{~cm}$ (diferencia de $9.78 \mathrm{~cm}$ ) y en mujeres de $58.5 \pm 8.7 \mathrm{~cm}$ a $67.23 \pm 9.1 \mathrm{~cm}$ (diferencia de $8.78 \mathrm{~cm}$ ).

La proporción de niños de seis y 12 años con sobrepeso fue de 11.2 y $9 \%$ y con obesidad, de 14.7 y $15 \%$, respectivamente. La proporción de niñas de seis y 12 años con sobrepeso fue de 8.2 y $9.1 \%$ y con obesidad de 21.7 y $13.3 \%$, respectivamente.

El promedio de la diferencia de talla de niños en edad escolar de Ponte al 100 al realizar la comparación con las tablas del doctor Ramos Galván (Figura 2a) fue inexistente, $-0.02 \mathrm{~m}(-0.04,0.01$, $\mathrm{p}=0.16)$; al realizar la comparación con las de los CDC (Figura 2b) fue de $-0.05 \mathrm{~m}(-0.07,-0.03$, $p<0.0001)$. En el caso de las niñas, en la comparación con las tablas del doctor Ramos Galván (Figura 2c) de igual forma fue prácticamente inexistente, aunque estadísticamente significativo: $-0.02 \mathrm{~m}(-0.04$, $0.00, p=0.02)$; en la comparación con las tablas de los CDC (Figura 2d) fue de $-0.05 \mathrm{~m}(-0.07,-0.04$, $p=0.00001)$.

\section{Discusión}

La progresión del peso y la talla de una población depende de diversos factores, tanto genéticos como

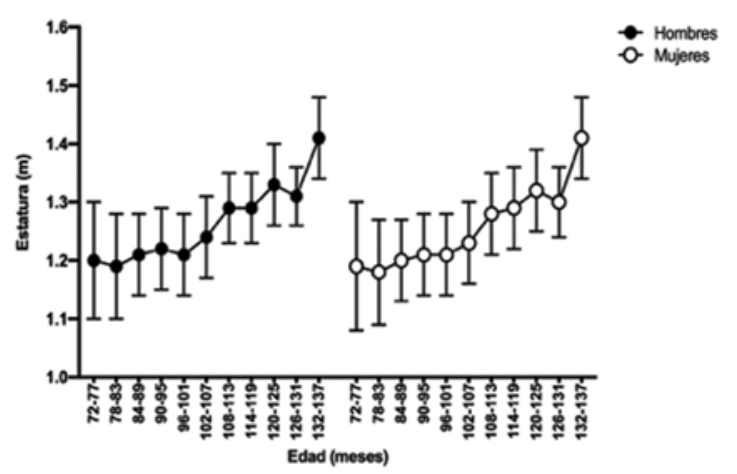

Figura 1. Talla medida en 43670 niños y 44103 niñas. La progresión de talla entre los 6 y 12 años fue de $21 \mathrm{~cm}$ en los hombres $(1.20 \pm 0.10$ a $1.41 \pm 0.07)$ y de $22 \mathrm{~cm}$ en las mujeres ( $1.19 \pm 0.11$ a $1.41 \pm 0.07)$.

ambientales. ${ }^{15} \mathrm{~A}$ nivel individual, la talla está genéticamente predeterminada por la funcionalidad e integridad de sistemas biológicos,$^{15}$ sin embargo, estos sistemas dependen del estado nutricional y de salud general del individuo. El incremento secular de la talla es un fenómeno en el que la estatura promedio de una población aumenta en función de transiciones demográficas y epidemiológicas, siendo un reflejo de las mejores condiciones de salud, nutricionales y socioeconómicas. ${ }^{16,17}$ El incremento secular de la talla durante el siglo XX está bien documentado en poblaciones europeas y de Estados Unidos. ${ }^{16-18}$ En México, este fenómeno ha sido identificado y caracterizado en poblaciones urbanas y rurales de Oaxaca. ${ }^{19,20}$

Nuestros resultados al inicio de la edad escolar (seis años) muestran un incremento de $4.5 \mathrm{~cm}$ en hombres (120 contra $115.5 \mathrm{~cm}$ ) y $4 \mathrm{~cm}$ en mujeres (115 contra $119 \mathrm{~cm}$ ), mayor que los referidos por las tablas de los CDC del año 2000..$^{21} \mathrm{~A}$ partir de los siete años estas diferencias desaparecen: la talla es $5 \mathrm{~cm}$ mayor en las referidas por la CDC y prácticamente la misma que la referida por el doctor Ramos Galván, ${ }^{8}$ por lo que no parece haber una ganancia significativa en los últimos 40 años. Desde el punto de vista de la talla a los siete años, nuestros resultados son similares a los reportados en otros países latinoamericanos como Colombia, ${ }^{6}$ Venezuela ${ }^{22}$ y Argentina, ${ }^{23}$ aunque a los 10 años en mujeres y a los 12 años en hombres fue menor. Cabe aclarar, sin embargo, que dichas series, particularmente la de Colombia incluye solamente individuos de clase socioeconómica alta, lo cual sin duda influye en el crecimiento y desarrollo de la población.

Los anteriores resultados hacen pensar que el desarrollo de talla en niños mexicanos sanos es igual en cualquier parte de la población y que no ha cambiado en los últimos 40 años, ya sea en la Ciudad de México, 
Tabla 1. Tabla de crecimiento en niños en edad escolar

\begin{tabular}{|c|c|c|c|c|c|c|c|c|c|}
\hline $\begin{array}{l}\text { Edad } \\
\text { en años } \\
\text { (rango en } \\
\text { meses) }\end{array}$ & $\begin{array}{c}\text { Ponte al } \\
100^{*} \\
\text { Media } \pm \mathrm{DE} \\
\text { (rango) }\end{array}$ & $\begin{array}{c}\text { Ramos } \\
\text { Galván* } \\
\text { Media } \pm \text { DE } \\
\text { (n) }\end{array}$ & $\begin{array}{c}\text { CDC }^{*} \\
\text { Media } \pm \text { DE } \\
\text { (n) }\end{array}$ & $\begin{array}{l}\text { Peso, kg } \\
\text { Media } \pm \mathrm{DE} \\
\text { (rango) }\end{array}$ & $\begin{array}{c}\mathrm{IMC}, \mathrm{kg} / \mathrm{m}^{2} \\
\text { Media } \pm \mathrm{DE} \\
\text { (rango) }\end{array}$ & $\begin{array}{l}\% \text { de grasa } \\
\text { Media } \pm \mathrm{DE}\end{array}$ & $\begin{array}{l}\text { Perímetro de } \\
\text { cintura, cm } \\
\text { Media } \pm \mathrm{DE}\end{array}$ & $\begin{array}{l}\text { Sobrepeso, } \\
\text { CDC \% }\end{array}$ & $\begin{array}{l}\text { Obesidad, } \\
\text { CDC } \%\end{array}$ \\
\hline $\begin{array}{l}6 \text { años } \\
(n=525) \\
(72-77)\end{array}$ & $\begin{array}{l}1.20 \pm 0.10 \\
(0.96-1.48)\end{array}$ & $\begin{array}{c}1.14 \pm 0.04 \\
(60)\end{array}$ & $\begin{array}{c}1.17 \pm 0.05 \\
(700)\end{array}$ & $\begin{array}{c}25.62 \pm 6.87 \\
(16-47)\end{array}$ & $\begin{array}{l}17.1 \pm 2.7 \\
(9.6-31.5)\end{array}$ & $17.05 \pm 8.2$ & $58.41 \pm 8.02$ & 11.2 & 14.7 \\
\hline $\begin{array}{l}6 \text { años y } 6 \\
\text { meses } \\
(n=1111) \\
(78-83)\end{array}$ & $\begin{array}{l}1.19 \pm 0.09 \\
(0.97-1.48)\end{array}$ & $\begin{array}{c}1.14 \pm 0.04 \\
(60)\end{array}$ & $\begin{array}{c}1.20 \pm 0.05 \\
(700)\end{array}$ & $\begin{array}{c}24.25 \pm 5.87 \\
(15.2-47)\end{array}$ & $\begin{array}{c}16.8 \pm 2.9 \\
(9.7-47)\end{array}$ & $16.88 \pm 7.9$ & $56.91 \pm 7.19$ & 9.7 & 8.8 \\
\hline $\begin{array}{l}7 \text { años } \\
(n=2538) \\
(84-89)\end{array}$ & $\begin{array}{l}1.21 \pm 0.07 \\
(1.05-1.40)\end{array}$ & $\begin{array}{c}1.20 \pm 0.04 \\
(57)\end{array}$ & $\begin{array}{c}1.23 \pm 0.05 \\
(700)\end{array}$ & $\begin{array}{c}24.43 \pm 4.71 \\
(15.2-38.9)\end{array}$ & $\begin{array}{l}16.5 \pm 2.3 \\
(8.5-32.2)\end{array}$ & $18.79 \pm 8.5$ & $57.42 \pm 7.26$ & 9.0 & 14.6 \\
\hline $\begin{array}{l}7 \text { años y } 6 \\
\text { meses } \\
(n=4350) \\
(90-95)\end{array}$ & $\begin{array}{l}1.22 \pm 0.07 \\
(1.05-1.40)\end{array}$ & $\begin{array}{c}1.23 \pm 0.05 \\
(57)\end{array}$ & $\begin{array}{c}1.26 \pm 0.05 \\
(700)\end{array}$ & $\begin{array}{c}24.74 \pm 4.78 \\
(15.1-38.9)\end{array}$ & $\begin{array}{l}16.4 \pm 2.2 \\
(8.1-27.9)\end{array}$ & $18.45 \pm 8.7$ & $57.61 \pm 7.47$ & 9.1 & 13.8 \\
\hline $\begin{array}{l}8 \text { años } \\
(n=7071) \\
(96-101)\end{array}$ & $\begin{array}{l}1.21 \pm 0.07 \\
(1.07-1.40)\end{array}$ & $\begin{array}{c}1.26 \pm 0.05 \\
(55)\end{array}$ & $\begin{array}{c}1.29 \pm 0.06 \\
(700)\end{array}$ & $\begin{array}{c}24.46 \pm 4.89 \\
(15.2-38.7)\end{array}$ & $\begin{array}{l}16.4 \pm 2.3 \\
(8.6-30.3)\end{array}$ & $17.28 \pm 8.5$ & $57.41 \pm 7.04$ & 9.3 & 11.5 \\
\hline $\begin{array}{l}8 \text { años y } 6 \\
\text { meses } \\
(n=4339) \\
(102-107)\end{array}$ & $\begin{array}{l}1.24 \pm 0.07 \\
(1.07-1.40)\end{array}$ & $\begin{array}{c}1.28 \pm 0.05 \\
(55)\end{array}$ & $\begin{array}{c}1.32 \pm 0.06 \\
(700)\end{array}$ & $\begin{array}{c}25.24 \pm 4.89 \\
(15.9-38.7)\end{array}$ & $\begin{array}{l}16.3 \pm 2.2 \\
(8.1-28.9)\end{array}$ & $16.71 \pm 8.5$ & $57.90 \pm 7.05$ & 9.2 & 13.4 \\
\hline $\begin{array}{l}9 \text { años } \\
(n=5166) \\
(108-113)\end{array}$ & $\begin{array}{l}1.29 \pm 0.06 \\
(1.01-1.45)\end{array}$ & $\begin{array}{c}1.33 \pm 0.05 \\
(52)\end{array}$ & $\begin{array}{c}1.35 \pm 0.05 \\
(700)\end{array}$ & $\begin{array}{c}28.55 \pm 5.66 \\
(17-43.3)\end{array}$ & $\begin{array}{c}17.1 \pm 2.6 \\
(9.4-33)\end{array}$ & $17.33 \pm 8.7$ & $60.34 \pm 7.77$ & 9.1 & 13.6 \\
\hline $\begin{array}{l}9 \text { años y } 6 \\
\text { meses } \\
(n=2799) \\
(114-119)\end{array}$ & $\begin{array}{l}1.29 \pm 0.06 \\
(1.14-1.45)\end{array}$ & $\begin{array}{c}1.33 \pm 0.05 \\
(52)\end{array}$ & $\begin{array}{c}1.37 \pm 0.07 \\
(700)\end{array}$ & $\begin{array}{l}28.1 \pm 5.3 \\
(16.3-43.3)\end{array}$ & $\begin{array}{l}16.6 \pm 2.2 \\
(8.5-31.7)\end{array}$ & $16.51 \pm 8.5$ & $59.83 \pm 7.04$ & 9.2 & 12.2 \\
\hline $\begin{array}{l}10 \text { años } \\
(n=6799) \\
(120-125)\end{array}$ & $\begin{array}{l}1.33 \pm 0.07 \\
(1.17-1.49)\end{array}$ & $\begin{array}{c}1.36 \pm 0.06 \\
(40)\end{array}$ & $\begin{array}{c}1.40 \pm 0.07 \\
(700)\end{array}$ & $\begin{array}{c}31.78 \pm 6.82 \\
(17.3-48.9)\end{array}$ & $\begin{array}{l}17.8 \pm 2.9 \\
(9.0-33.9)\end{array}$ & $17.85 \pm 8.6$ & $62.88 \pm 8.49$ & 9.0 & 13.9 \\
\hline $\begin{array}{l}10 \text { años } 6 \\
\text { meses } \\
(n=1179) \\
(126-131)\end{array}$ & $\begin{array}{l}1.31 \pm 0.05 \\
(1.17-1.45)\end{array}$ & $\begin{array}{c}1.36 \pm 0.06 \\
(40)\end{array}$ & $\begin{array}{c}1.42 \pm 0.07 \\
(700)\end{array}$ & $\begin{array}{c}29.13 \pm 4.82 \\
(18-47)\end{array}$ & $\begin{array}{l}16.9 \pm 2.4 \\
(9.8-28.2)\end{array}$ & $16.03 \pm 7.9$ & $59.97 \pm 6.08$ & 9.8 & 7.6 \\
\hline $\begin{array}{l}11 \text { años } \\
(n=7793) \\
(132-137)\end{array}$ & $\begin{array}{l}1.41 \pm 0.07 \\
(1.25-1.56)\end{array}$ & $\begin{array}{c}1.41 \pm 0.06 \\
(34)\end{array}$ & $\begin{array}{c}1.45 \pm 0.07 \\
(700)\end{array}$ & $\begin{array}{c}35.48 \pm 7.33 \\
(19.5-54.6)\end{array}$ & $\begin{array}{l}17.9 \pm 2.8 \\
(9.1-31.2)\end{array}$ & $17.58 \pm 7.9$ & $68.18 \pm 9.57$ & 9.0 & 15 \\
\hline
\end{tabular}

*Talla en metros. DE = desviación estándar estimada, CDC = Centers for Disease Control.

como lo mostraron las curvas del doctor Ramos Galván hace más de 40 años, o en el resto del país, como lo muestran nuestros resultados, en los que están representadas poblaciones urbanas, suburbanas y rurales. Las tablas del doctor Ramos Galván incluyeron predominantemente niños sanos de la Ciudad de México, que resultaron ser una muestra adecuada, ya que para esa época era la zona de mayor desarrollo socioeconómico del país, lo que garantizaba una buena alimentación y mostraba el desarrollo pleno de la talla. Cuarenta años después, podemos observar que el desarrollo promedio de la talla ha alcanzado su plenitud como resultado de una mejoría en el acceso a alimentos y a servicios de salud a lo largo del territorio mexicano. ${ }^{24,25}$

Es importante resaltar que nuestra población representa a niños sanos en edad escolar de escuelas públicas de todo el país. ${ }^{13}$ La prevalencia de obesidad 
Tabla 2. Tabla de crecimiento en niñas en edad escolar

\begin{tabular}{|c|c|c|c|c|c|c|c|c|c|}
\hline $\begin{array}{l}\text { Edad } \\
\text { en años } \\
\text { (rango en } \\
\text { meses) }\end{array}$ & $\begin{array}{l}\text { Ponte al } \\
100^{*} \\
\text { Media } \pm \mathrm{DE} \\
\text { (rango) }\end{array}$ & $\begin{array}{c}\text { Ramos } \\
\text { Galván* } \\
\text { Media } \pm \text { DE } \\
\text { (N) }\end{array}$ & $\begin{array}{c}\text { CDC }^{*} \\
\text { Media } \pm \text { DE } \\
\text { (n) }\end{array}$ & $\begin{array}{l}\text { Peso, kg } \\
\text { Promedio } \\
\text { (rango) }\end{array}$ & $\begin{array}{c}\text { IMC, } \mathrm{kg} / \mathrm{m}^{2} \\
\text { Promedio } \\
\text { (rango) }\end{array}$ & $\begin{array}{l}\% \text { de grasa } \\
\text { Media } \pm \mathrm{DE}\end{array}$ & $\begin{array}{l}\text { Perímetro de } \\
\text { cintura, cm } \\
\text { Media } \pm \mathrm{DE}\end{array}$ & $\begin{array}{l}\text { Sobrepeso } \\
\text { (CDC) \% }\end{array}$ & $\begin{array}{l}\text { Obesidad } \\
\text { (CDC) \% }\end{array}$ \\
\hline $\begin{array}{l}6 \text { años } \\
(n=475) \\
(72-77)\end{array}$ & $1.19 \pm 0.11$ & $\begin{array}{c}1.14 \pm 0.04 \\
(118)\end{array}$ & $\begin{array}{c}1.16 \pm 0.07 \\
(700)\end{array}$ & $\begin{array}{c}25.42 \pm 6.63 \\
(16-48)\end{array}$ & $\begin{array}{l}17.4 \pm 3.4 \\
(7.2-42.4)\end{array}$ & $19.5 \pm 7.9$ & $58.5 \pm 8.7$ & 8.2 & 21.7 \\
\hline $\begin{array}{l}6 \text { año y } 6 \\
\text { meses } \\
(n=1124) \\
(78-83)\end{array}$ & $\begin{array}{l}1.18 \pm 0.09 \\
(0.98-1.49)\end{array}$ & $\begin{array}{c}1.17 \pm 0.05 \\
(118)\end{array}$ & $\begin{array}{l}1.20 \pm 0.05 \\
(700)\end{array}$ & $\begin{array}{c}24.05 \pm 5.95 \\
(15-48)\end{array}$ & $\begin{array}{c}16.8 \pm 3 \\
(8.6-35.1)\end{array}$ & $19.2 \pm 7.2$ & $56.41 \pm 7.24$ & 9.4 & 9.6 \\
\hline $\begin{array}{l}7 \text { años } \\
(n=2473) \\
(84-89)\end{array}$ & $\begin{array}{l}1.20 \pm 0.07 \\
(1.04-1.40)\end{array}$ & $\begin{array}{c}1.20 \pm 0.05 \\
(130)\end{array}$ & $\begin{array}{l}1.23 \pm 0.05 \\
(700)\end{array}$ & $\begin{array}{c}24.01 \pm 4.84 \\
\quad(15.3-39)\end{array}$ & $\begin{array}{l}16.4 \pm 2.5 \\
(8.8-31.4)\end{array}$ & $21.11 \pm 7.3$ & $56.92 \pm 7.17$ & 9.1 & 13.5 \\
\hline $\begin{array}{l}7 \text { años y } 6 \\
\text { meses } \\
(n=4362) \\
(90-95)\end{array}$ & $\begin{array}{l}1.21 \pm 0.07 \\
(1.04-1.40)\end{array}$ & $\begin{array}{c}1.22 \pm 0.05 \\
(130)\end{array}$ & $\begin{array}{c}1.26 \pm 0.06 \\
(700)\end{array}$ & $\begin{array}{l}24.54 \pm 5 \\
(15.1-39)\end{array}$ & $\begin{array}{l}16.5 \pm 2.4 \\
(9.6-35.1)\end{array}$ & $20.99 \pm 7.3$ & $57.47 \pm 7.58$ & 7.2 & 14.7 \\
\hline $\begin{array}{l}8 \text { años } \\
(n=6984) \\
(96-101)\end{array}$ & $\begin{array}{l}1.21 \pm 0.07 \\
(1.06-1.39)\end{array}$ & $\begin{array}{c}1.25 \pm 0.05 \\
(152)\end{array}$ & $\begin{array}{c}1.29 \pm 0.06 \\
(700)\end{array}$ & $\begin{array}{l}24.07 \pm 4.9 \\
(15.5-38.2)\end{array}$ & $\begin{array}{l}16.3 \pm 2.3 \\
(9.8-29.7)\end{array}$ & $19.96 \pm 7.0$ & $56.96 \pm 7.10$ & 9.2 & 11.8 \\
\hline $\begin{array}{l}8 \text { años y } 6 \\
\text { meses } \\
(n=4261) \\
(102-107)\end{array}$ & $\begin{array}{l}1.23 \pm 0.07 \\
(1.06-1.09)\end{array}$ & $\begin{array}{c}1.28 \pm 0.05 \\
(152)\end{array}$ & $\begin{array}{c}1.31 \pm 0.06 \\
(700)\end{array}$ & $\begin{array}{c}24.84 \pm 4.97 \\
(16-38.2)\end{array}$ & $\begin{array}{l}16.3 \pm 2.3 \\
(9.9-32.5)\end{array}$ & $19.77 \pm 7.0$ & $57.62 \pm 7.08$ & 9.2 & 12.9 \\
\hline $\begin{array}{l}9 \text { años } \\
(n=5259) \\
(108-113)\end{array}$ & $\begin{array}{l}1.28 \pm 0.07 \\
(1.13-1.53)\end{array}$ & $\begin{array}{c}1.30 \pm 0.05 \\
(153)\end{array}$ & $\begin{array}{c}1.34 \pm 0.06 \\
(700)\end{array}$ & $\begin{array}{c}28.09 \pm 5.58 \\
(17-42.7)\end{array}$ & $\begin{array}{l}17.0 \pm 2.5 \\
(8.8-32.8)\end{array}$ & $20.86 \pm 7.2$ & $59.99 \pm 7.48$ & 9.2 & 12.4 \\
\hline $\begin{array}{l}9 \text { años y } 6 \\
\text { meses } \\
(n=2918) \\
(114-119)\end{array}$ & $\begin{array}{l}1.29 \pm 0.07 \\
(1.13-1.53)\end{array}$ & $\begin{array}{c}1.33 \pm 0.06 \\
(153)\end{array}$ & $\begin{array}{c}1.36 \pm 0.06 \\
(700)\end{array}$ & $\begin{array}{c}27.86 \pm 5.31 \\
(17-42.7)\end{array}$ & $\begin{array}{l}16.6 \pm 2.1 \\
(8.8-29.9)\end{array}$ & $20.32 \pm 7.1$ & $59.43 \pm 7.06$ & 9.3 & 10.4 \\
\hline $\begin{array}{l}10 \text { años } \\
(n=6850) \\
(120-125)\end{array}$ & $\begin{array}{l}1.32 \pm 0.07 \\
(1.16-1.48)\end{array}$ & $\begin{array}{c}1.36 \pm 0.06 \\
(143)\end{array}$ & $\begin{array}{c}1.39 \pm 0.07 \\
(700)\end{array}$ & $\begin{array}{c}31.27 \pm 6.92 \\
(17.2-48.3)\end{array}$ & $\begin{array}{l}17.5 \pm 2.8 \\
(8.9-31.4)\end{array}$ & $21.48 \pm 7.0$ & $62.08 \pm 8.2$ & 9.0 & 15.0 \\
\hline $\begin{array}{l}\text { 10años y } 6 \\
\text { meses } \\
(n=1308) \\
(126-131)\end{array}$ & $\begin{array}{l}1.30 \pm 0.06 \\
(1.16-1.48)\end{array}$ & $\begin{array}{c}1.39 \pm 0.06 \\
(143)\end{array}$ & $\begin{array}{c}1.42 \pm 0.09 \\
(700)\end{array}$ & $\begin{array}{c}29.08 \pm 5.08 \\
(18-48)\end{array}$ & $\begin{array}{l}16.9 \pm 2.6 \\
(9.1-32.1)\end{array}$ & $19.7 \pm 6.4$ & $59.78 \pm 6.5$ & 9.7 & 8.0 \\
\hline $\begin{array}{l}11 \text { años } \\
(n=8089) \\
(132-137)\end{array}$ & $\begin{array}{l}1.41 \pm 0.07 \\
(1.23-1.57)\end{array}$ & $\begin{array}{c}1.43 \pm 0.07 \\
(141)\end{array}$ & $\begin{array}{c}1.45 \pm 0.07 \\
(700)\end{array}$ & $\begin{array}{c}35.47 \pm 7.56 \\
(20-54.3)\end{array}$ & $\begin{array}{l}17.8 \pm 2.8 \\
(9.9-32.7)\end{array}$ & $20.33 \pm 7.0$ & $67.23 \pm 9.1$ & 9.1 & 13.3 \\
\hline
\end{tabular}

*Talla en metros, DE = desviación estándar estimada, CDC = Centers for Desease Control.

encontrada en esta población a diferentes edades (de 7.6 a $15 \%$ en niños y de 8 a $21 \%$ en niñas) es similar a la reportada en ENSANUT 2016 de Medio Camino, lo que indica claramente la representatividad nacional de nuestra población. ${ }^{26}$ La transición epidemiológica muestra el paso poblacional de la desnutrición a un peso adecuado para la edad y la talla, pero pasando en forma inevitable por el sobrepeso y la obesidad. Es de esperar que conforme continúen mejorando las condiciones de nutrición del país se observe una reducción en la incidencia de sobrepeso y obesidad como consecuencia de una mejora en el acceso a alimentos de mejor calidad, acompañada de mejores hábitos de actividad física, acciones que se promueven en programas como Ponte al 100.13,27

Entre las limitaciones de nuestro análisis está la posible variabilidad en las mediciones como resultado del número de evaluadores, aunque todos tenían un 


\begin{tabular}{|c|c|c|c|c|c|c|c|c|c|c|}
\hline \multirow{2}{*}{$\frac{\text { Grupo }}{6 \text { años }}$} & \multicolumn{2}{|c|}{$\begin{array}{c}\text { Ponte al } 100 \\
\text { Media }[\mathrm{m}] \quad \mathrm{DE}[\mathrm{m}]\end{array}$} & Total & \multicolumn{3}{|c|}{ Ramos Galván } & Peso & \multirow{2}{*}{$\begin{array}{l}\begin{array}{l}\text { Diferencia de medias } \\
\text { Aleatorio IC 95\% [m] }\end{array} \\
0.06[0.05,0.07]\end{array}$} & $\begin{array}{l}\text { Diferencia de medias } \\
\text { Aleatorio IC } 95 \%[\mathrm{~m}]\end{array}$ & \\
\hline & 1.2 & 0.1 & 525 & 1.14 & 0.04 & 60 & $9.2 \%$ & & & \\
\hline 6 años 6 meses & 1.19 & 0.09 & 1111 & 1.17 & 0.04 & 60 & $9.3 \%$ & $0.02[0.01,0.03]$ & & \\
\hline 7 años & 1.21 & 0.07 & 2538 & 1.2 & 0.04 & 57 & $9.3 \%$ & $0.01[-0.00,0.02]$ & & \\
\hline 7 años 6 meses & 1.22 & 0.07 & 4350 & 1.23 & 0.05 & 57 & $9.2 \%$ & $-0.01[-0.02,0.00]$ & & \\
\hline 8 años & 1.21 & 0.07 & 7071 & 1.26 & 0.05 & 55 & $9.2 \%$ & $-0.05[-0.06,-0.04]$ & & \\
\hline 8 años 6 meses & 1.24 & 0.07 & 4339 & 1.28 & 0.05 & 55 & $9.2 \%$ & $-0.04[-0.05,-0.03]$ & & \\
\hline 9 años & 1.29 & 0.06 & 5166 & 1.33 & 0.05 & 52 & $9.2 \%$ & $-0.04[-0.05,-0.03]$ & & \\
\hline 9 años 6 meses & 1.29 & 0.06 & 2799 & 1.33 & 0.05 & 52 & $9.1 \%$ & $-0.04[-0.05,-0.03]$ & & \\
\hline 10 años & 1.33 & 0.07 & 6799 & 1.36 & 0.06 & 40 & $8.9 \%$ & $-0.03[-0.05,-0.01]$ & & \\
\hline 10 años 6 meses & 1.31 & 0.05 & 1179 & 1.36 & 0.06 & 40 & $8.9 \%$ & $-0.05[-0.07,-0.03]$ & & \\
\hline 11 años & 1.41 & 0.07 & 7793 & 1.41 & 0.06 & 34 & $8.8 \%$ & $0.00[-0.02,0.02]$ & & \\
\hline Total IC $95 \%$ & & & 43670 & & & 562 & $100.0 \%$ & $-0.02[-0.04,0.01]$ & & \\
\hline $\begin{array}{l}\text { Heterogeneidad } \\
\text { Prueba para efectos }\end{array}$ & $\begin{array}{l}\mathrm{Tau}^{2}=0.00 \\
\text { totales } \quad \mathrm{Z}=1\end{array}$ & $\begin{array}{l}h i^{2}= \\
10(p=\end{array}$ & $\begin{array}{l}260.21, \\
=0.16)\end{array}$ &,$g l=10(p<$ & 0.0000 & $; 1^{2}=$ & $96 \%$ & & $\begin{array}{ccc}-0.05 & 0 & 0.02 \\
\text { Ramos Galvan } & \text { Ponte }\end{array}$ & $\begin{array}{l}0.05 \\
100\end{array}$ \\
\hline
\end{tabular}

b

\begin{tabular}{|c|c|c|c|c|c|c|c|c|c|c|c|}
\hline Grupo & \multicolumn{3}{|c|}{ Ponte al 100} & \multicolumn{3}{|c|}{ CDC } & Peso & $\begin{array}{l}\text { Diferencia de medias } \\
\text { Aleatorio IC } 95 \%[\mathrm{~m}]\end{array}$ & \multicolumn{3}{|c|}{$\begin{array}{l}\text { Diferencia de medias } \\
\text { Aleatorio IC } 95 \%[\mathrm{~m}]\end{array}$} \\
\hline 6 años & 1.2 & 0.1 & 525 & 1.17 & 0.05 & 700 & $9.0 \%$ & $0.03[0.02,0.04]$ & & & -- \\
\hline 6 años 6 meses & 1.19 & 0.09 & 1111 & 1.2 & 0.05 & 700 & $9.1 \%$ & $-0.01[-0.02,-0.00]$ & & - & \\
\hline 7 años & 1.21 & 0.07 & 2538 & 1.23 & 0.05 & 700 & $9.1 \%$ & $-0.02[-0.02,-0.02]$ & & - & \\
\hline 7 años 6 meses & 1.22 & 0.07 & 4350 & 1.26 & 0.05 & 700 & $9.1 \%$ & $-0.04[-0.04,-0.04]$ & & - & \\
\hline 8 años & 1.21 & 0.07 & 7071 & 1.29 & 0.06 & 700 & $9.1 \%$ & $-0.08[-0.08,-0.08]$ & - & & \\
\hline 8 años 6 meses & 1.24 & 0.07 & 4339 & 1.32 & 0.06 & 700 & $9.1 \%$ & $-0.08[-0.08,-0.08]$ & - & & \\
\hline 9 años & 1.29 & 0.06 & 5166 & 1.35 & 0.05 & 700 & $9.1 \%$ & $-0.06[-0.06,-0.06]$ & & $=$ & \\
\hline 9 años 6 meses & 1.29 & 0.06 & 2799 & 1.37 & 0.07 & 700 & $9.1 \%$ & $-0.08[-0.09,-0.07]$ & - & & \\
\hline 10 años & 1.33 & 0.07 & 6799 & 1.4 & 0.07 & 700 & $9.1 \%$ & $-0.07[-0.08,-0.06]$ & & - & \\
\hline 10 años 6 meses & 1.31 & 0.05 & 1179 & 1.42 & 0.07 & 700 & $9.1 \%$ & $-0.11[-0.12,-0.10]$ & - & & \\
\hline 11 años & 1.41 & 0.07 & 7793 & 1.45 & 0.07 & 700 & $9.1 \%$ & $-0.04[-0.05,-0.03]$ & & - & \\
\hline Total IC $95 \%$ & & & 43670 & & & 7700 & $100.0 \%$ & $-0.05[-0.07,-0.03]$ & & & \\
\hline $\begin{array}{l}\text { Heterogeneidad } T \\
\text { Prueba para efecto }\end{array}$ & $\begin{array}{l}=0.00 ; \mathrm{Cr} \\
\text { otales } \quad \mathrm{Z}\end{array}$ & $\begin{array}{r}=145 \\
5.29(p\end{array}$ & $\begin{array}{l}4.08, \mathrm{gl} \\
<0.000\end{array}$ & $\begin{array}{l}=10(p< \\
01)\end{array}$ & $0001)$ & $; 1^{2}=99$ & & & -0.1 & $5 \mathrm{CDC}$ & $\begin{array}{c}0.05 \\
\text { Ponte al } 10\end{array}$ \\
\hline
\end{tabular}

c

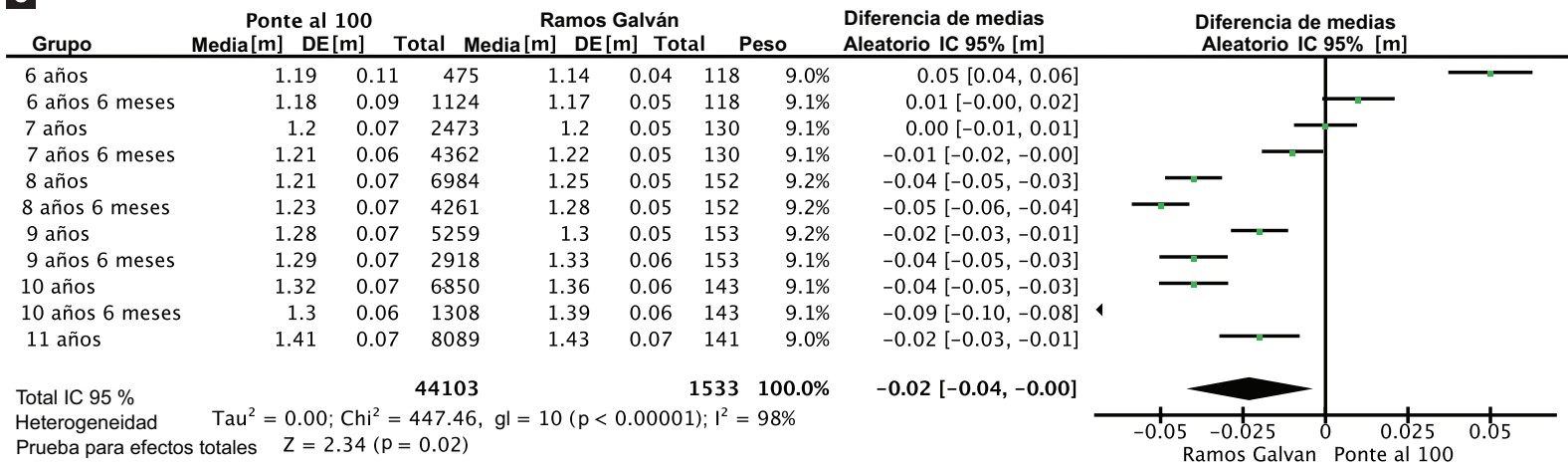

d

\begin{tabular}{|c|c|c|c|c|c|c|c|c|c|c|c|}
\hline Grupo & \multicolumn{2}{|c|}{$\begin{array}{r}\text { Ponte al } 100 \\
\text { Media }[\mathrm{m}] \text { DE }[\mathrm{m}]\end{array}$} & \multirow{2}{*}{$\frac{\text { Total }}{475}$} & \multicolumn{2}{|c|}{$\begin{array}{l}\mathrm{CDC} \\
\text { 1] } \mathrm{DE}\end{array}$} & Total & Peso & $\begin{array}{l}\text { Diferencia de medias } \\
\text { Aleatorio IC } 95 \%[\mathrm{~m}]\end{array}$ & \multicolumn{3}{|c|}{$\begin{array}{l}\text { Diferencia de medias } \\
\text { Aleatorio IC } 95 \%[\mathrm{~m}]\end{array}$} \\
\hline 6 años & 1.19 & 0.11 & & 1.16 & 0.07 & 700 & $8.8 \%$ & $0.03[0.02,0.04]$ & & & 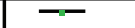 \\
\hline 6 años 6 meses & 1.18 & 0.09 & 1124 & 1.2 & 0.05 & 700 & $9.1 \%$ & $-0.02[-0.03,-0.01]$ & & - & \\
\hline 7 años & 1.2 & 0.07 & 2473 & 1.23 & 0.05 & 700 & $9.1 \%$ & $-0.03[-0.03,-0.03]$ & & - & \\
\hline 7 años 6 meses & 1.21 & 0.06 & 4362 & 1.26 & 0.07 & 700 & $9.1 \%$ & $-0.05[-0.06,-0.04]$ & & $=$ & \\
\hline 8 años & 1.21 & 0.07 & 6984 & 1.29 & 0.06 & 700 & $9.1 \%$ & $-0.08[-0.08,-0.08]$ & & - & \\
\hline 8 años 6 meses & 1.23 & 0.07 & 4261 & 1.31 & 0.06 & 700 & $9.1 \%$ & $-0.08[-0.08,-0.08]$ & & - & \\
\hline 9 años & 1.28 & 0.07 & 5259 & 1.34 & 0.06 & 700 & $9.1 \%$ & $-0.06[-0.06,-0.06]$ & & 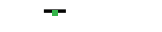 & \\
\hline 9 años 6 meses & 1.29 & 0.07 & 2918 & 1.36 & 0.06 & 700 & $9.1 \%$ & $-0.07[-0.08,-0.06]$ & & - & \\
\hline 10 años & 1.32 & 0.07 & 6850 & 1.39 & 0.07 & 700 & $9.1 \%$ & $-0.07[-0.08,-0.06]$ & & - & \\
\hline 10 años 6 meses & 1.3 & 0.06 & 1308 & 1.42 & 0.09 & 700 & $9.0 \%$ & $-0.12[-0.13,-0.11]$ & $*$ & & \\
\hline 11 años & 1.41 & 0.07 & 8089 & 1.45 & 0.07 & 700 & $9.1 \%$ & $-0.04[-0.05,-0.03]$ & & - & \\
\hline Total IC $95 \%$ & & & 44103 & & & 7700 & $100.0 \%$ & $-0.05[-0.07,-0.04]$ & & & \\
\hline $\begin{array}{l}\text { Heterogeneidad } \\
\text { Prueba para efecto }\end{array}$ & $\begin{array}{c}\operatorname{Tau}^{2}=0 \\
\text { otales } \\
Z\end{array}$ & $\begin{array}{l}0 ; \mathrm{Chi}^{2}= \\
6.23(\mathrm{p}\end{array}$ & $\begin{array}{l}=1032 \\
<0.00\end{array}$ & $\begin{array}{l}71, \mathrm{gl}=10 \\
01)\end{array}$ & $<0.0$ & 0001); & $2=99 \%$ & & & & $\begin{array}{r}0.05 \\
\text { al } 10\end{array}$ \\
\hline
\end{tabular}

Figura 2. Promedios de la diferencia de talla de niños y niñas en edad escolar registrados en el programa Ponte al 100. a) Niños, $-0.02 \mathrm{~m}$ $(-0.04,0.01, p=0.16)$ en comparación con las tablas doctor Ramos Galván. b) Niños, $-0.05 m(-0.07,-0.03, p<0.0001)$ en comparación con las tablas de la CDC. c) Niñas, $-0.02 m(-0.04,0.00, p=0.02)$ en comparación con las tablas del doctor Ramos Galván. $d)$ Niñas, $-0.05 m$ $(-0.07,-0.04, p=0.00001)$ en comparación con las tablas de la CDC.

nivel similar de escolaridad y fueron estandarizados en las técnicas. Entre las ventajas, está el tamaño de la muestra, más de 87000 niños y niñas, por lo que se trata del estudio antropométrico más grande del mundo, ${ }^{5,6,28-31}$ además de incluir todas las zonas geográficas y estratos socioeconómicos de México. 
Aun cuando el incremento secular de la talla a través de los siglos es un fenómeno documentado, ${ }^{17}$ en nuestro estudio no logramos identificarlo. Una explicación es que como especie hemos alcanzado la talla máxima, cuyo promedio se mantiene estable debido a la optimización en el acceso a alimentos y servicios de salud. Alternativamente existe la posibilidad de que se requiera más tiempo de seguimiento para identificar un verdadero incremento secular de la talla.

Existen múltiples cuestionamientos por contestar, entre otros evaluar si en los niños obesos el crecimiento es más acelerado. El reto como país es y seguirá siendo en los siguientes años el acceso a alimentos de mayor calidad y el desarrollo de hábitos de actividad física que conlleven a mejoría en la capacidad funcional y la calidad de vida. ${ }^{32-34}$

\section{Bibliografía}

1. Bann D, Johnson W, Li L, Kuh D, Hardy R. Socioeconomic inequalities in childhood and adolescent body-mass index, weight, and height from 1953 to 2015: an analysis of four longitudinal, observational, British birth cohort studies. Lancet Public Health. 2018;3:e194-e203.

2. Gonçalves H, Barros FC, Buffarini R, Horta BL, Menezes AMB Barros AJD, et al. Infant nutrition and growth: trends and inequalities in four population-based birth cohorts in Pelotas, Brazil, 1982-2015. Int J Epidemiol. 2019;48:i80-i88.

3. Manios Y, Karatzi K, Moschonis G, loannou G, Androutsos O, Lionis C, et al. Lifestyle, anthropometric, socio-demographic and perinatal correlates of early adolescence hypertension: The Healthy Growth Study. Nutr Metab Cardiovasc Dis. 2019;29:159-169.

4. Loret de Mola C, Quispe R, Valle GA, Poterico JA. Nutritional transition in children under five years and women of reproductive age: a 15-years trend analysis in Peru. PLoS One. 2014;9:e92550.

5. Cardoso HF, Padez C. Changes in height, weight, BMI and in the prevalence of obesity among 9-to-11-year-old affluent Portuguese schoolboys, between 1960 and 2000. Ann Hum Biol. 2008;35:624-638.

6. Durán $\mathrm{P}$, Merker A, Briceño G, Colón E, Line D, Abad V, et al. Colombian reference growth curves for height, weight, body mass index and head circumference. Acta Pediatr. 2016;105:e116-e125.

7. Bustamante A, Freitas D, Pan H, Katzmarzyk PT, Maia J. Centile curves and reference values for height, body mass, body mass index and wais circumference of Peruvian children and adolescents. Int J Environ Res Public Health. 2015;12:2905-2922.

8. Ramos-Galván R. Pediatric somatometry. Semilongituginal study of children in Mexico City. Arch Invest Med (Mex). 1975;6:83-396.

9. Erdei G, Bakacs M, Ilés É, Nagy B, Kaposvári C, Mák E, et al. Substantial variation across geographic regions in the obesity prevalence among 6-8 years old Hungarian children (COSI Hungary 2016). BMC Public Health. 2018;18:611.

10. Márquez-Gonzáles H, García-Sámano VM, Caltenco-Serrano ML, García-Villegas EA, Márquez-Flores H, Billa-Romero AR. Clasificación y evaluación de la desnutrición en el paciente pediátrico. El Residente. 2010;7:59-69.

11. Rito $A$, Wijnhoven $T M$, Rutter $H$, Carvalho MA, Paixão $E$, Ramos $C$, et al. Prevalence of obesity among Portuguese children (6-8 years old) using three definition criteria: COSI Portugal, 2008. Pediatr Obes. 2012;7:413-422.
12. Brito-Zurita OR, López-Leal J, Exiga-González EB, Armenta-Llanes O, Jorge-Plascencia B, Domínguez-Banda A, et al. Medidas antropométricas. en la población infantil urbana de 6 a 12 años del noroeste de México. Rev Med Inst Mex Seguro Soc. 2014;52:S34-S41.

13. Palacios-Butchart $\mathrm{JJ}$, Herrera-Navarro JM, Melgar V Rangel MJ, Ferreira-Hermosillo A, Roy-García I, et al. Ponte al 100: a nationwide exercise and nutrition intervention program in Mexican children and adolescents: study population and methodology. Rev Mex Endocrinol Metab Nutr. 2016;3:175-181.

14. Rivas-Ruiz R, Castelán-Martínez OD, Pérez-Rodríguez M, Palacios-Cruz L, Noyola-Castillo ME, Talavera JO. Clinical research XXIII. From clinical judgment to meta-analyses. Rev Med Inst Mex Seguro Soc. 2014:52:558-65.

15. Benyi E, Sävendahl L. The physiology of childhood growth: hormonal regulation. Horm Res Paediatr. 2017;88:6-14.

16. Cole TJ. Secular trends in growth. Proc Nutr Soc. 2000;59:317-324

17. Fudvoye J, Parent AS. Secular trends in growth. Ann Endocrinol (Paris). 2017;78:88-91.

18. Meredith HV. Findings from Asia, Australia, Europe and North America on secular change in mean height of children youths and young adults. Am J Phys Anthropol. 1976:44:315-325.

19. Malina RM Little BB, Peña-Reyes ME. Secular trends are associated with the demographic and epidemiologic transitions in an indigenous community in Oaxaca, Southern Mexico. Am J Phys Anthropol. 2018:165:47-64.

20. Malina RM, Peña-Reyes ME, Little BB. Secular change in the growth status of urban and rural school children aged 6-13 years in Oaxaca, southern Mexico. Ann Hum Biol. 2008:35:475-489.

21. Kuczmarski RJ, Ogden CL, Guo SS, Grummer-Strawn LM, Flegal KM, Mei Z, et al. 2000 CDC growth charts for the United States: methods and development. Vital Health Stat 11. 2002;246:1-190.

22. López-Blanco M, Landaeta-Jiménez M, Izagirre-Espinoza I, Macías-Tomei C. Estudio nacional de crecimiento y desarrollo humano en la República de Venezuela. Venezuela: Escuela Técnica Salesiana; 1996.

23. Lejarraga $H$, Orfila $G$. Weight and height standards for Argentinian girls and boys from birth to maturity. Arch Argent Pediatr. 1987:85:209-22.

24. Turnbull B, Gordon SF, Martínez-Andrade GO, González-Unzaga M. Childhood obesity in Mexico: a critical analysis of the environmental factors, behaviors and discourses contributing to the epidemic. Health Psychol Open. 2019;6:2055102919849406.

25. Vásquez-Garibay EM, Miranda-Ríos L, Romero-Velarde E, Nuño-Cosío ME, Campos-Barrera L, Nápoles-Rodríguez F, et al. Stunting, overweight and obesity during the nutrition transition in schoolchildren of Arandas, Jalisco, Mexico. Rev Med Inst Mex Seguro Soc. 2018;56:6-11.

26. Encuesta Nacional de Salud y Nutrición de Medio Camino 2016. Informe final de resultados. México: Instituto Nacional de Salud Pública; 2016.

27. Urquía-Fernández N. La seguridad alimentaria en México. Salud Publica Mex. 2014;56:S92-S98.

28. Orden AB, Torres MF, Castro L, Cesani MF, Luis MA, Quintero FA, et al. Physical growth in schoolchildren from Argentina: comparison with Argentinean and CDC/NCHs growth references. Am J Hum Biol. 2009;21:312-318.

29. Garzón M, Papoila AL, Alves M, Pereira-da-Silva L. Comparison of growth curve estimates of infants in São Tomé Island, Africa, with the WHO growth standards: a birth cohort study. Int J Environ Res Public Health. 2019;16:E1693.

30. El Mouzan MI, Shaffi A, Salloum AA, Alqurashi MM, Herbish AA Omer AA. Z-score growth reference data for Saudi preschool children. Ann Saudi Med. 2017;37:10-15.

31. Neyzi O, Bundak R, Gökçay G, Günöz H, Furman A, Darendeliler F, et al. Reference Values for weight, height, head circumference, and body mass index in Turkish children. J Clin Res Pediatr Endocrinol. 2015;7:280-293.

32. Wen CP, Wai JP, Tsai MK, Yang YC, Cheng TY, Lee MC, et al. Minimum amount of physical activity for reduced mortality and extended life expectancy: a prospective cohort study. Lancet. 2011;378:1244-1253.

33. Redondo-Tébar A, Ruiz-Hermosa A, Martínez-Vizcaíno V, Cobo-Cuenca Al, Bermejo-Cantarero A, Cavero-Redondo I, et al. Associations between health-related quality of life and physical fitness in 4-7-year-old Spanish children: the MOVIKIDS study. Qual Life Res. 2019;28:1751-1759.

34. Oja P, Kelly P, Pedisic Z, Titze S, Bauman A, Foster C, et al. Associations of specific types of sports and exercise with all-cause and cardiovascular-disease mortality: a cohort study of 80306 British adults. $\mathrm{Br} \mathrm{J}$ Sports Med. 2017;51:812-817. 